\title{
E-business Ecosystem and its Evolutionary Path: \\ The Case of the Alibaba Group in China
}

\author{
Lihua Huang \\ Fudan University \\ 670 Guoshun Road, Shanghai 200433, China \\ lhhuang@fudan.edu.cn \\ Ganglan Hu \\ Fudan University \\ 670 Guoshun Road, Shanghai 200433, China \\ 071025001@fudan.edu.cn \\ Xianghua Lu \\ Fudan University \\ 670 Guoshun Road, Shanghai 200433, China \\ Ixhua@fudan.edu.cn
}

\begin{abstract}
The rapid growth of online consumers and e-business websites in China has attracted a large number of organizations to participate in the e-business industry; many of them have gone through several stages of evolution and aggregations. The purpose of this study is to apply the business ecosystem theory as the theoretical lens to explore the phenomenon of e-business growth in China. The Alibaba Group was chosen as a case to demonstrate the process by which a typical e-business ecosystem got shaped, developed, and matured. Four stages in the evolution path of Alibaba's ecosystem and their respective characteristics are identified. The findings are useful for understanding the development of e-business in china. Implications for researchers and practitioners are discussed.
\end{abstract}

Keywords: E-business evolution, e-Marketplace, Business ecosystem, Alibaba Group 


\section{Introduction}

The application of e-business has grown rapidly in China in the past decade. The number of netizens shopping online, the amount of online transactions, and the variety of e-business websites increase significantly, primarily due to the concerted efforts of the government in developing the infrastructure necessary for the Internet utilization (Raven et al., 2004). Based on a survey conducted by iResearch, online retail transactions in 2007 has a year-on-year increase of nearly $100 \%$ and account for $0.64 \%$ of China's total consumption. This online consumption ratio is expected to grow to $5 \%$ in 5 years (iResearch, 2008).

The development of the online market and ebusiness websites attracts more organizations to participate in e-businesses. These organizations emerge to gather around a few keystone e-business companies to enhance their competitive advantage and achieve economies of scale. For example, large e-business companies such as Ctrip.com, Alibaba Group, and Tencent.com have been surrounded by a large number of related organizations, including merchandise providers (e.g., retailers, manufacturers, and individual sellers), service providers (e.g., logistic companies, software houses, financial institutions), government agencies, and value-added service providers such as certification agencies, internet-personnel training and education institutions.

Many researchers have paid attention to the rapid growth of e-business in China, and quite a few e-commerce companies are listed in Nasdaq and are well-valued. For example, the stock price of Baidu.com was over US $\$ 700$ before its split. However, few studies have emphasized on the ecosystem of these e-business companies and other organizations surrounding them. It is, therefore, interesting to explore the issues such as "how and why are these companies and organizations form an aggregation", or "What's the characteristic of these e-business clusters". In this paper, we use the Alibaba Group, the largest e-commerce company in
China, as our target to investigate its growth from a start-up company to a big enterprise. The business ecosystem theory is adopted as the theoretical lens for our study, because the theory can better capture the characteristics of e-business industry in China.

This paper is structured as follows. Section 2 briefly introduces the theoretical background of the study. Section 3 describes our research design, followed by the description of the Alibaba.com case and our observations in Sections 4 and 5. Finally, the paper is concluded in Section 6.

\section{Theoretical background}

The study was based on two key concepts: business ecosystem and e-business ecosystem, which are introduced in this section.

\section{Business ecosystem theory}

In 1993, the notion "business ecosystem" was first introduced by Moore in his paper "Predator and Prey: A New Ecology of Competition" published in Harvard Business Review. In that paper, he divided the evolution of a business ecosystem into four distinct stages: birth, expansion, leadership, and self-renewal (Moore, 1993). A business ecosystem can be conceived as a network of interdependent niches occupied by organizations that work cooperatively and competitively to support new products, satisfy customer needs, and eventually incorporate the next round of innovations (Moore, 2006). Companies are viewed not as a member of a single industry but as part of a business ecosystem that crosses a variety of industries.

The business ecosystem theory covers a range beyond the general industry. It describes the relationship between enterprises and environment (Dobson, 2006). Currently, e-businesses in China show the phenomenon of a common client-oriented and symbiotically-related aggregation, with the similar characteristics as a business ecosystem in many aspects, including features, elements, structure and functions. Compared with other competition theories such as the value chain theory or dynamic 
alliance theory, the business ecosystem theory considers more factors and components (e.g., rivals and government institutions), stresses more on the coexistence between players, and gives more attention on its evolution process and the adaptation capacity to the environment (Gossain and Kandiah, 1998; lansiti and Richard, 2006). The business ecosystem theory can better explain the aggregation of relevant companies and organizations in the Chinese e-business industry.

\section{Electronic business ecosystem}

Based on the business ecosystem theory, we can define the phenomenon of e-business aggregation (called the electronic business ecosystem or e-business ecosystem in short) as 'an organic ecosystem that is made of enterprises and organizations with close relations, using the internet as a platform to make competition and communication through virtual alliance, sharing resources, and making full use of their advantages beyond geographic limits'. In a welldeveloped e-business ecosystem, all members have their functions to form a value network with the flow of materials, energy and information.

A typical e-business ecosystem is composed of the following 4 different categories of companies and institutions:

- Lead Species: This is the core ebusiness of the ecosystem. It provides the business platform and regulatory services to serve the role of resource integration and coordination for the ecosystem.

- Key Species: They are the customers of the other species in the e-business ecosystem and main players involved in e-business trading, including consumers, retailers, manufacturers, special suppliers, and etc..

- $\quad$ Supportive Species: These species make the ecosystem more efficient but are not dependent on the e-business ecosystem for their survival. They can gain more benefits from the optimized e-business ecosystem than that from their own competition (Nguyen, 2002). They are indispensable companies and organizations in the e-business ecosystem, including logistics companies, financial institutions, telecommunication suppliers, government agencies, and so on.

- Parasitic Species: These species are companies that must coexist with the ebusiness ecosystem. They rely on the ecosystem for survival. Typical instances are value-added service providers, such as technology vendors, advertising and marketing service providers, training agencies and consulting firms.

Figure 1 shows the relationship and dependence of these species in an ebusiness ecosystem.

A typical business ecosystem can go through four evolutionary stages: birth, expansion, coordination and maturity, and death. At the birth stage, e-business enterprises view certain specific group of customers as target, attracting participants via innovative operation model and high value products or services, creating and emerging a new e-business ecosystem. Some ecosystems that capture the needs of the customers will survive at this stage and develop further into a larger scale.

At the expansion stage, as the development of the Lead species, the number of key species and supportive species keeps increasing and the parasitical species gradually emerge. While e-business ecosystem expands to conquer broad new territories at this stage, the competition with other ecosystem becomes more intensified. At the coordination and maturity stage, the relationships between various species become increasingly complicated due to the rapid growth of all the species at their expansion stage, especially, the competitions and conflicts among key species and parasitic species for the interests. To some extent, lead species need to regulate and perfect the system rules to maintain the healthy development of the system at the maturity stage. 
The evolution stage occurs when a mature business ecosystem is threatened by rising new ecosystems or external environment changes. The ecosystem may either evolving into a new ecosystem by completely changing the original business model or declining and fading away, as the eBay ecosystem decided to pull out the China market Figure 2 shows the evolutionary path graphically.

Using the business ecosystem theory as the lens, this study attempts to answer two research questions: "How have e-business ecosystems in China been formed?" and "What characterize the e-business ecosystem in China?"

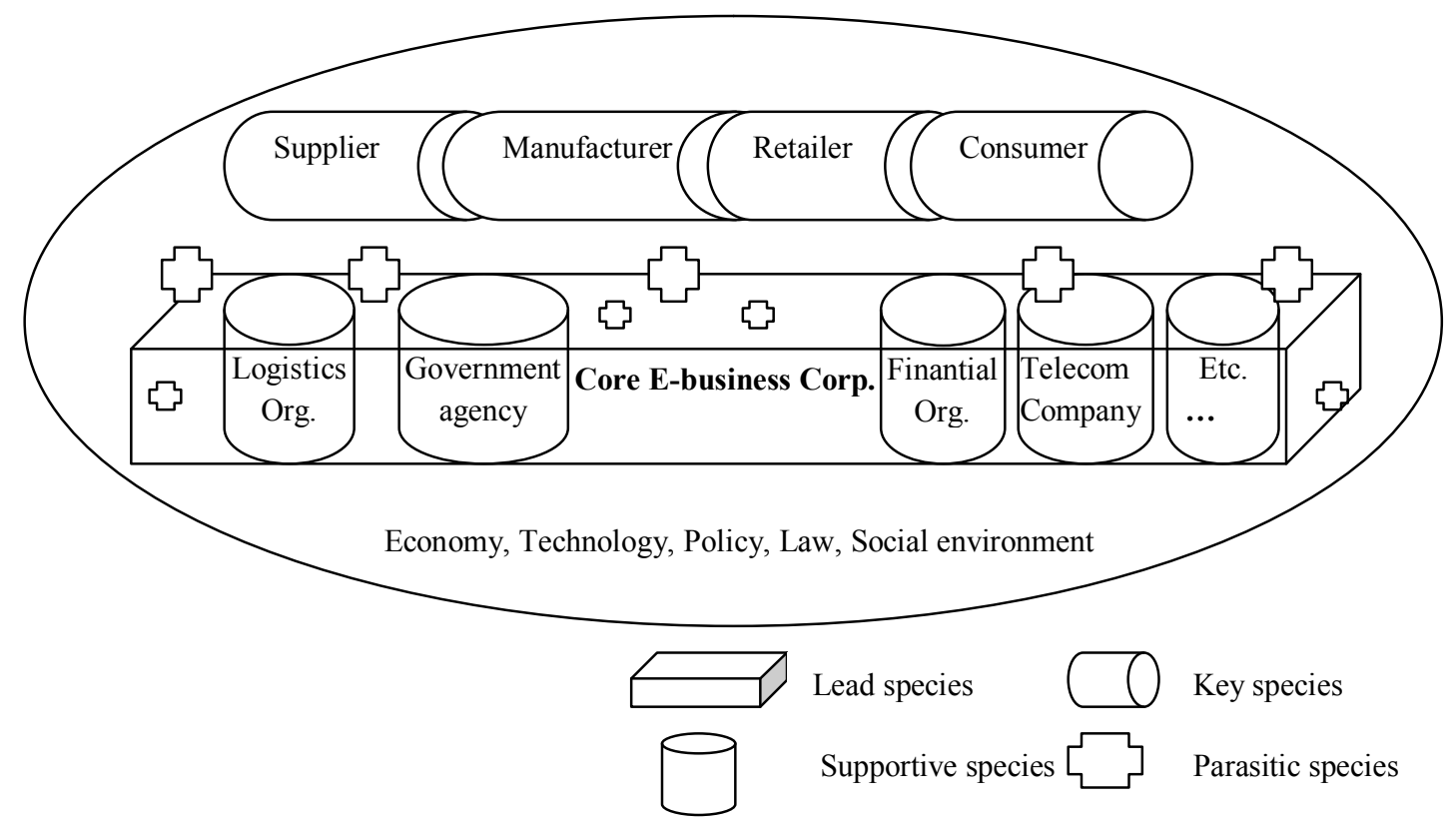

\section{Figure 1 - Elements of an e-business ecosystem}

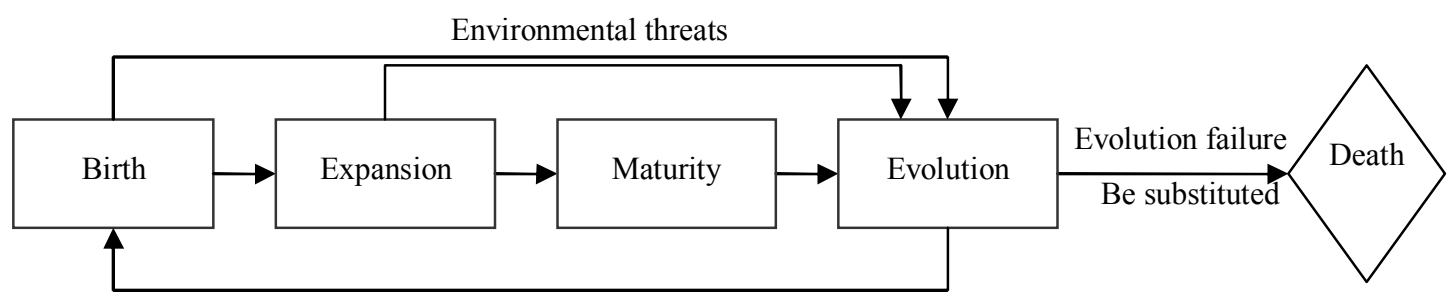

A New E-business Ecosystem

\section{Figure 2 - The Evolutionary Path of a Business Ecosystem}




\section{Research Design}

The case method was chosen for this research, mainly because our research questions are "how" and "why" questions (Walsham, 1995) that delves into the underlying process through which the aggregations in e-business industry are formed and developed. This is a phenomenon that cannot be easily quantified. Furthermore, the case method is more suitable for the longitudinal nature of this study.

The Alibaba.com was chosen as the case for our study based on two criteria. First, the selected organization must be the core of a well-developed and representative ebusiness ecosystem and be surrounded by a large number of associated organizations. Second, it should have a reasonably long history, which makes it possible to analyze the evolutionary process. The case of Alibaba Group met both criteria: the Alibaba Group was the largest e-business group in China and it had gone through 10-years' evolution.

The Alibaba Ecosystem has been well developed and played an important role in China's e-business industry. The use of a single case study for our research is also advantageous in that many contextual variables are kept constant, which helps to rule out possible alternative interpretations of the data.

The data was collected over a period of two months, from the middle of July 2008 to the middle of September 2008. Unstructured interviews and second-hand material were used for data collection. Open-ended questions for interview and data gathering forms were prepared before the interview sessions. These questions were carefully designed to gather information about the whole development process of Alibaba Group and its associated partners. The interviewees were asked to answer all questions based on their knowledge. A total of 10 interviews were conducted and the participants were selected based on their roles and their long-term working experience with Alibaba Group. They include 1 vice president of Alibaba Group, 7 senior managers in 5 subsidiary companies, and 2 VIP users of the company's main website.

In addition to interviews, second-hand data were collected from the company's document and the Internet. Data analysis, performed in tandem with data collection, was carried out by moving back and forth between empirical data, relevant literature and the emerging theory (Walsham, 2006; Eisenhardt, 1989). The researchers used coding and constantcomparison methods to analyze the collected data (Carver, 2004).

\section{Evolution of Alibaba}

\section{Organizational Background}

The Alibaba Group was established in 1999 and had become a global e-business leader and the largest e-business group in China at the time we conducted the research. Alibaba Group had reached the Internet users in more than 240 regions, and employed 17,000 people in more than 50 cities in Greater China, Japan, Korea, the United Kingdom and the United States.

Alibaba Group initially provided efficient and convenient business-to-business (B2B) online service for small and medium-size enterprises (SMEs), while it had grown to include 5 subsidiary companies of different businesses: online global leading B2B trading platform (Alibaba.com), China's largest online commercial centre (Taobao), third-party online payment services (Alipay), one of China's leading Internet portals (China Yahoo!), and developer of advanced datacentric computing services (Alibaba Cloud Computing). The history and milestones of Alibaba Group is summarized in Table 1. 


\begin{tabular}{|c|c|}
\hline 1999 & $\begin{array}{l}\text { Alibaba Group is officially established by its } 18 \text { founders, led by Jack Ma, working out of a } \\
\text { Hangzhou apartment. }\end{array}$ \\
\hline $1999-2000$ & $\begin{array}{l}\text { Alibaba Group raises US } \$ 25 \text { million from Softbank, Goldman Sachs, Fidelity, and other } \\
\text { institutions. }\end{array}$ \\
\hline 2002 & Alibaba.com becomes profitable. \\
\hline 2003 & Consumer e-commerce website Taobao is founded, again in Jack Ma's apartment. \\
\hline 2004 & Online payment system Alipay is launched. \\
\hline 2005 & $\begin{array}{l}\text { Alibaba Group forms a strategic partnership with Yahoo! Inc. and takes over the operation of } \\
\text { China Yahoo!. }\end{array}$ \\
\hline 2006 & Alibaba Group makes a strategic investment in Koubei.com. \\
\hline 2007 & $\begin{array}{l}\text { Internet-based business software company Alisoft is launched. (January) } \\
\text { Alibaba.com Limited lists on the Hong Kong Stock Exchange. (November) } \\
\text { Alibaba Group launches Alimama, an online advertising exchange company. (November) }\end{array}$ \\
\hline 2008 & 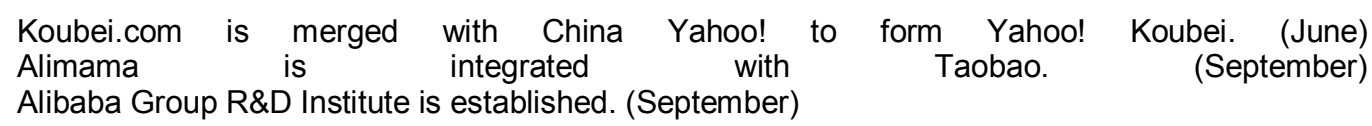 \\
\hline 2009 & $\begin{array}{l}\text { Alisoft is merged with Alibaba Group R\&D Institute. (July) } \\
\text { Alisoft's Business Management Software division is injected into Alibaba.com. (August) } \\
\text { Koubei.com is injected into Taobao as part of the "Big Taobao" strategy, which positions Taobao } \\
\text { as a one-stop e-commerce service provider to promote wider use of e-commerce among } \\
\text { consumers. } \\
\text { Alibaba Cloud Computing is established in conjunction with Alibaba Group's 10th anniversary } \\
\text { celebration. (September) }\end{array}$ \\
\hline 2010 & $\begin{array}{l}\text { Alibaba Group creates a cross-business team comprising senior managers from Taobao, Alipay, } \\
\text { Alibaba Cloud Computing and China Yahoo! to execute a full-scale roll-out of the "Big Taobao" } \\
\text { strategy. } \\
\text { (March) } \\
\text { Alibaba Group announces that it will begin in } 2010 \text { to earmark } 0.3 \text { percent of annual revenues to } \\
\text { fund efforts designed to spur environmental awareness and conservation in China and around the } \\
\text { world. (May) }\end{array}$ \\
\hline
\end{tabular}

\section{Evolution of Alibaba.com}

Through the interviews, we found that Alibaba Group has attracted a large number of institutes to involve and the number was continuously increasing during the past 10 years. It had evolved from a unitary functioned e-business website to lead species of an e-business ecosystem, circled by a large number of buyers and sellers (key species), logistics corporations, financial institutions, telecommunication providers, government agencies (supportive species), and value-added service providers (parasitic species).
A typical life cycle of business ecosystem includes four stages: birth, expansion, maturity, and evolution or death. After analyzing the collected data, we found that the Alibaba Ecosystem had experienced 3 main stages of evolution: birth, expansion, and maturity. Another stage, the evolution stage, is predicted.

\section{Birth stage (1999 - 2002)}

Alibaba Ecosystem started its exploration in 1999 as a B2B e-marketplace, when SMEs were lack of trading opportunities. By the integration of relative resources, Alibaba Group built an Internet-based trading platform 
to reduce the cost of marketing and information search for SMEs. Therefore, the major environmental driver for the formation of the company was the rapid growth of SMEs in China and their strong need for a trading channel to access the world.

At this stage, the ecosystem had a very simple structure, including Alibaba.com as the core, users as the key participants, and some other essential supportive species. The core Alibaba Group also had to perform functions which should have been provided by supportive and parasitic species. A manager described the status of the ecosystem around 2000:

"Internet in China was not fully developed at that time. Not many people are willing to trade online... Door-to-door marketing was required. Besides, the technical facilities were not complete enough to support safe and convenient e-business... Many necessary functions were provided by our own."

Yet, through the large-scale updating, trial-toerrors, and improvement, Alibaba Ecosystem had transitioned from a single functional trading forum to a trading platform offering spirited new products and services, including China Suppliers and Trust Pass. The ecosystem successfully survived the major threat of Internet bubble burst. The major reason that Alibaba was able to survive the Internet bubble burst was the rich resource provided by the environment - many western companies were looking for inexpensive suppliers in China. The economic recession might have further boosted that need.

\section{Expansion stage (2003 - 2007)}

In fact, the increasing need in Developed countries for outsourcing their manufacturing to China helped Alibaba not only survived the Internet bubble but also started its expansion afterward. The boundary of the ecosystem was broadened rapidly, and the participants had been continuously and dramatically increasing. Many new ideas were adopted and implemented quickly. They might disappear quickly after proven to be no value either. The ecosystem's high updating frequency and the character of vague boundary began to appear up. One senior manager indicated as follows:

"We began to expand since 2003, starting from moving into online personal trading $(\mathrm{C} 2 \mathrm{C})$. In the following 4 years, our service extended from ordinary goods trading to online software and advertisement business, and from goods trading market to life service community... Our strategies were to try and change. Ideas did not need to fully mature before trying to implement. Environmental change was too fast, we had to update before competitors and environmental changes... Changes happened everyday."

Meanwhile, the competition between Alibaba and other business ecosystems became fierce when it entered different markets. The most well-known was the competition with the eBay China ecosystem in the online auction market. The war ended with Ebay.com's withdrawal from the China market, which resulted in Alibaba Group's C2C business to be the Number 1 leader in China.

There are many reasons that have caused the success of Alibaba (or the failure of eBay). A major one is the lack of cultural sensitivity of eBay. As indicated by an interviewee, eBay copied the whole western model from its headquarter to its acquired subsidiary company (which was the largest auction website in China before the acquisition). Unfortunately, the key species of ebay ecosystem forced other members to follow its culture which did not fit the business environment in China. For example, eBay didn't allow buyers and sellers to communicate before the transaction, which was quite common in western countries. This rule was not acceptable in Chins. As an old Chinese proverb says, "friendship first, business follows," communication and emotional involvement were critical to successful trading in China at least in the online market at that time when trust among trading partners was pretty weak. Additionally, 
buyers in China are used to bargaining with the sellers before the purchase. Unwilling to follow the local culture and adapting its strategy accordingly had costed the eBay ecosystem its market share in China and eventually gave the market to Alibaba's Taobao.com (the Chinese meaning of "finding treasury"). A senior manager of Taobao.com elaborated that:

"The competition with eBay could not be avoided. We had similar target markets and customers... The reason we won the battle is due to two main magic weapons: Tool and Service. We provided communication tools between buyers and sellers and a better thirdparty payment intermediary service... More importantly, all these general services were free."

In addition to the successful entry of the C2C market, Alibaba also acquired China Yahoo! in 2005. Overall, the Alibaba ecosystem expanded its coverage into different markets, including $\mathrm{C} 2 \mathrm{C}$ online auction and e-payment markets. It successfully defeated competitors in those markets through strategies that better fitted the local environment - the trading behaviour of customers.

\section{Coordination and Maturity stage (2008 - 2009)}

With the fast growth of Alibaba ecosystem, its members increase substantially in a short time period, it is quickly entering the maturity stage. Alibaba was listed in the Hong Kong Stock Exchange in 2007. Its strategies started to be under scrutiny of professional investors and the general public.

The growth of the ecosystem also needs more coordination among its members. As the members increase, the interests among these participants become more diversified and are conflicting sometimes. The ecological rules that have guided the growth of the ecofamily may not meet the present needs and environmental challenges. The company started two major strategic moves: one was to improve its coordination for high effectiveness and another was to enhance its technological infrastructure to meet new trends. From the strategic side, it changed the previous sub-company strategy to the "Big Taobao Strategy", that will switch the core species of the ecosystem from the original e-business service provider to a new e-business infrastructure operator. The core species will serve as an independent platform operator that does not engage in any specific e-business trading any more. The ultimate goal of this strategic transformation is to strengthen the openness of the ecosystem, to standardize various services, in order to attract more environmental resources and to achieve sustainable development of the whole ecosystem. This opening strategy may also reduce the conflict among different species in the ecosystem.

Another change is that the group started a cloud computing company to cope with new technological trends. The new technological platform may be able to facilitate the "Big Taobao" strategy and avoid potential new entrants who will take advantage of the cloud computing technology to invade into the market.

\section{Evolution in the future}

Although the Alibaba Group is in a healthy situation, any substantial external threat could subvert the stability of the ecosystem. The VP of Alibaba Group indicated in the interview that
"The company is aware of the potential crisis in our daily operation. To us, potential competitors could be someone in a college dormitory right now, who would overthrow our current business model and be involved in e-business with an incredibly new model."

Thus, Within the Alibaba Group, an important business is to assess the value of its affiliated partners. Termination of business without competitiveness and creation of new initiatives are of high priority. They recognize that the best solution when facing fatal external threats is to withdraw from declining markets and capture new business opportunities in advance to ensure the 
sustainability of the ecosystem. The evolution stage is ahead, which could result in death or a brand-new more competitive ecosystem.

\section{Discussion}

From the interview and analysis of the Alibaba case, we have found a few characteristics of e-business ecosystems, which makes it different from the traditional business ecosystem.

First, a business ecosystem is highly dynamic. Traditional business ecosystem only experiences a large degree of member changes in the last phase of the whole evolutionary path. In the Alibaba ecosystem, however, we found that member updates continued to occur throughout the process of birth, expansion and maturity. We believed that the high updating rate in e-business ecosystem could be resulted from ebusiness's two essential principles: trial-toerror and innovation.

Second, the leadership of the core ebusiness enterprise is critical and stable. In a traditional business ecosystem, different central companies could use their bargaining powers to seek the leadership in the ecosystem at the maturity stage. But, in the Alibaba case, we found that the dominance of Alibaba Group is quite immune to the challenge of other core companies. It is believed that the network effect could account for the steady leadership of the core enterprise in the e-business ecosystem. The Internet promises an exponential increase in value for core enterprises that are connecting with a large number of clients, which will in turn appeal more potential customers. As a result, the network effect will drive the power to be even stronger, and win the high loyalty of customers, due to their habituation to the products and services offered by the lead enterprises.

Third, the boundary of the ecosystem is vague. Traditional business ecosystems are usually restricted to one specific area, while the Alibaba ecosystem has continuously extended its boundaries to various areas, including product review, forums and virtual communities, life service information, search engines, and so on. This may also due to the characteristics of the Internet, which include the low cost of information sharing and no geographical nor time restrictions in access. The open access feature provides the ebusiness ecosystem with the ability to derive other trade-related services.

Fourth, the environmental threat is constantly high. For traditional business ecosystems, decline occurs only when mature business communities are threatened by rising new ecosystems and innovations at the stage of evolution. However, the Alibaba ecosystem shows that the e-business ecosystem has faced higher uncertainty and environmental threats throughout the whole development process, due to lack of standard business models, and continuous changes in technology and government policies. For example, imposing tax on online transactions or prohibiting online imitation has direct impact on the competition in the e-business ecosystem. Therefore, decline and death could happen more often in different stages of the evolution as compared with traditional business ecosystems.

To summarize, the development of Alibaba ecosystem has indicated key characteristics in different stages of the e-business ecosystem. Alibaba Group, as the lead species of the e-business ecosystem, has played an irreplaceable role at every evolutionary stage. According to the case, we summarize in Table 2 the features of the ebusiness ecosystem at each stage, and the roles played and strategies taken by lead species at corresponding stage.

Because the development of e-business is still relatively young and unstable, an ebusiness ecosystem could suffer from the sudden change of its external environment, no matter at its birth, expansion, or coordination stage, and directly enter into its evolution stage. Or, we may argue that the evolutionary path of an e-business ecosystem is much shorter that that of a traditional ecosystem. 


\begin{tabular}{|l|l|l|}
\hline Table 2 - E-business Ecosystem Evolutionary Path \\
\hline Stage & Feature & Role of Core E-business Enterprise \\
\hline Birth Stage & $\begin{array}{l}\text { E-business } \\
\text { ecosystem creation }\end{array}$ & $\begin{array}{l}\text { Understand and catch key species' requirements, explore a new } \\
\text { e-business ecosystem which has special viability, and then } \\
\text { prompt its birth and make it take shape. }\end{array}$ \\
\hline $\begin{array}{l}\text { Expansion } \\
\text { Stage }\end{array}$ & $\begin{array}{l}\text { Species increasing } \\
\text { competition with other } \\
\text { ecosystem }\end{array}$ & $\begin{array}{l}\text { Absorb all available resources continuously, launch various } \\
\text { valuable products or services, and speed up the growth of other } \\
\text { species; Meanwhile, deal with the conflict with other } \\
\text { homogeneous ecosystem. }\end{array}$ \\
\hline $\begin{array}{l}\text { Coordination } \\
\text { Stage }\end{array}$ & $\begin{array}{l}\text { Intense conflicts } \\
\text { between internal } \\
\text { species, ecosystem } \\
\text { rules improvement }\end{array}$ & $\begin{array}{l}\text { Improve and perfect ecosystem's rules, coordinate and } \\
\text { supervise the other species, and then allow the ecosystem to a } \\
\text { healthy and harmonious development. }\end{array}$ \\
\hline Evolution Stage & $\begin{array}{l}\text { Overthrow of the } \\
\text { original e-business } \\
\text { ecosystem }\end{array}$ & $\begin{array}{l}\text { Cope with fatal threats produced by new technologies, new } \\
\text { models, or other change of external environment, explore any } \\
\text { possible outlets, and then overthrow the original ecosystem by } \\
\text { new technology, trading or consumption pattern, or profit rules. }\end{array}$ \\
\hline
\end{tabular}

\section{Conclusion}

This study has investigated the evolution of a major e-business ecosystem in China, the Alibaba Group. We have discovered major features in each stage of the evolutionary path. Our contributions are as follows. First, by describing how e-business companies and organizations gathered together, shaped an ecosystem, developed and matured to achieve better overall competitive advantage in the Alibaba ecosystem,, the paper provides valuable information about how an ebusiness ecosystem works in China.

Second, by applying the business ecosystem theory, this study presents a new perspective to understand the phenomenon of e-business. We introduce the concept of e-business ecosystem and argue that organizations using the Internet as a platform to establish virtual alliance, share resources, and make use of their advantages with no geographic limits can be analyzed from the ecological perspective to find more insight into their operations and competitive advantages.

Third, from the practical perspective, the Alibaba case demonstrates the importance of continuous updating, trial on different innovative ideas, and attention to customer needs and local cultures. More significantly, the analysis of e-business ecosystem members, evolutionary path, and unique characteristics is important for e-business companies to realize their position in the ecosystem and set up effective strategies at corresponding evolutionary stages. The findings are also useful for online companies who are interested in entering the China market.

As an exploratory research, the study is not without its limitations. A particular criticism that is commonly directed at studies using the case research methodology is the problem of generalizability. While it must be readily acknowledged that statistical generalization is impossible from a single case study, generalizations can take the form of concepts, theories, specific implications or rich insights (Walsham, 1995; 2006), and it is possible to generalize non-statistically from case description to theory, as in the case of this article, within a single case study (Lee and Baskerville, 2003). A second limitation is that this study observed the e-business ecosystem mainly from the perspective of the lead species and only interviewed the participants representing lead species and key species. More emphasis could be laid on the other two species to validate the results and get a better understanding of the complete picture. Nonetheless, the findings are valuable for those who are interested in opportunities and development of e-business in China. 


\section{References}

Alibaba Group, (2009), "Company overview", http://news.alibaba.com/specials/about alibaba/aligroup/index.html, sited on Nov., 16, 2009

Carver J., (2004), "Using Qualitative Methods in Software Engineering". Presented at International Advanced School of Empirical Software Engineering (IASESE), 18 August, Los Angeles, CA.

Dobson, P.W., (2006), "Competing, Countervailing and Coalescing Forces: the Economics of Intra- and Interbusiness System Competition", Antitrust Bulletin, 51 (1), pp.175-193.

Eisenhardt, K.M. (1989), "Building theories from case study research," Academy Manage Review, 14 (4), pp. 532-550

Gossain, S., and G. Kandiah, (1998), "Reinventing Value: the New Business Ecosystem", Strategy \& Leadership, 26 (5), pp.28-33.

lansiti, M., and G.L. Richard, (2006), "The Information Technology Ecosystem: Structure, Health and Performance", Antitrust Bulletin, 51 (1), pp.77-110.

iResearch, (2008), "2007-2008 China Online Shopping Research Report", http://down.iresearch.cn/Reports/Char ge/1161.html, sited on Nov.10, 2008

Lee, A.S. and Baskerville R.L., (2003), "Generalizing generalizability in information systems research", Information System Research, Vol.14, No.3, pp.221-243

Moore, J.F., (2006), "Business Ecosystems and the View from the Firm", Antitrust Bulletin, 51 (1), pp.31-75

Moore, J.F., (1993), "Predator and Prey: A New Ecology of Competition", Harvard Business Review, 71 (3), pp.75-86.

Nguyen, T.N., (2002), "The Ecology of Software: A Framework for the Investigation of Business-IT Integration", Journal of American Academy of Business, 2 (1), pp.7-11.

Peltoniemi, M., and E. Vuori, (2004), "Business Ecosystem as the New Approach to Complex Adaptive Business Environments", Frontiers of E-business Research, pp.267-281

Raven, P.V., Huang, X.Q., and Kim B.B., 2004, "E-business in Developing Countries: A Comparison of China and India", International Journal of EBusiness Research, 3 (1), pp.91-108.

Walsham G., (1995), "Interpretive case studies in IS research: Nature and method," European Journal of Information System, 4 (2), pp. 74-81.

Walsham G., (2006), "Doing interpretive research," European Journal of Information System, 15 (3), pp. 320330 


\section{About the Authors}

Lihua Huang is the professor of information systems at the Department of Information Management, School of Management, Fudan University. She received her Ph.D in MIS from the Fudan University. Her research interests include e-business, ERP and information system management. Her publications have appeared in Information and Management, Europe Journal of Information Systems, Journal of Electronic Commerce Research, Decision Support Systems, IEEE Transactions on Engineering Management, International Journal of Production Economics, Journal of Global Information Management et al. She consults with major companies in Shanghai and government departments of the $P R$ China. She is a co-chair of the program committee of Asia Pacific Conference on Information Systems 2004 and ICIS 2011.
Ganglan $\mathrm{Hu}$ is a $\mathrm{PhD}$ student at Fudan University. She will get her PhD in MIS in July 2010. Her research interests include ebusiness, information system management, and IT capability. Her publications have appeared in IEEE software, proceedings of 3rd conference on Risk Management \& Global e-Business, and so on.

Xianghua $\mathrm{Lu}$ is an associate professor of Management Information System at the School of management, Fudan University, Shanghai. She received her Ph.D in MIS from the Fudan University. Her research interests include E-commerce, information system strategy management and IT business value management. Her publications have appeared in Information and Management, Global Journal of Information Technology Management, International Journal of ECRM, International Conference of Information Systems. 